Review

\title{
THERANOSTICS: From Molecular Imaging Using Ga-68 Labeled Tracers and PET/CT to Personalized Radionuclide Therapy - The Bad Berka Experience
}

\author{
Richard P. Baum ${ }^{凶}$, Harshad R. Kulkarni \\ Department of Nuclear Medicine/ Center for PET/CT, Zentralklinik Bad Berka, ENETS Center of Excellence, Zentralklinik \\ Bad Berka, 99437 Bad Berka, Germany.
}

Corresponding author: Professor Dr. Richard P. Baum, Chairman and Clinical Director, Dept. of Nuclear Medicine / Center for PET/CT, ENETS Center of Excellence, Zentralklinik Bad Berka, 99437 Bad Berka, Germany. Tel. +49 3645852200 , Fax +49364585 3515, richard.baum@zentralklinik.de.

(C) Ivyspring International Publisher. This is an open-access article distributed under the terms of the Creative Commons License (http://creativecommons.org/ licenses/by-nc-nd/3.0/). Reproduction is permitted for personal, noncommercial use, provided that the article is in whole, unmodified, and properly cited.

Received: 2011.10.16; Accepted: 2011.12.02; Published: 2012.05.07

\begin{abstract}
The acronym THERANOSTICS epitomizes the inseparability of diagnosis and therapy, the pillars of medicine and takes into account personalized management of disease for a specific patient. Molecular phenotypes of neoplasms can be determined by molecular imaging with specific probes using positron emission tomography (PET), single photon emission computed tomography (SPECT), magnetic resonance imaging (MRI), or optical methods, so that the treatment is specifically targeted against the tumor and its environment. To meet these demands, we need to define the targets, ligands, coupling and labeling chemistry, the most appropriate radionuclides, biodistribution modifiers, and finally select the right patients for the personalized treatment. THERANOSTICS of neuroendocrine tumors (NETs) using Ga-68 labeled tracers for diagnostics with positron emission tomography/ computed tomography (PET/CT), and using Lu-I77 or other metallic radionuclides for radionuclide therapy by applying the same peptide proves that personalized radionuclide therapy today is already a fact and not a fiction.
\end{abstract}

Key words: THERANOSTICS, molecular imaging, personalized radionuclide therapy.

\section{INTRODUCTION}

The term theranostics epitomizes the inseparability of diagnosis and therapy, the pillars of medicine. In the context of nuclear medicine, it refers to the use of molecular targeting vectors (e.g. peptides) labeled either with diagnostic radionuclides (e.g. positron or gamma emitters), or with therapeutic radionuclides for diagnosis and therapy respectively of a particular disease, targeted specifically by the vector at its molecular level. Therefore molecular imaging and diagnosis of the disease can be effectively followed by personalized treatment utilizing the same molecular imaging vectors. One of the classic examples of theranostics is the use of Ga-68 labeled tracers, wherein the diagnosis using this generator-derived radionuclide can be effectively followed by the therapy using therapeutic radionuclides like Lu-177 and Y-90 labeled with the same tracer for personalized radionuclide therapy. Addition of two important facets namely individualized patient dosimetry by preor post therapeutic imaging, and assessment of therapy response using quantitative imaging by Ga-68 positron emission tomography/ computed tomography (PET/CT) fulfills the potential of personalized medicine.

Ga-68 is an easily-available generator-derived diagnostic trivalent radio metal with convenient la- 
beling characteristics. ${ }^{1} \mathrm{Ga}-68$ is prepared from a $\mathrm{TiO}_{2}$ based Ge-68/Ga-68 generator system, which has a half-life of 288 days. $^{2}$ A GMP compliant, fully automated click and start cassette-based synthesis system with easy handling is now available (EZAG, Berlin, Germany) for the daily routine production of Ga-68 labeled radiopharmaceuticals. Post-processing of Ge-68/Ga-68 radionuclide generators using cation exchange resin provides chemically and ra-

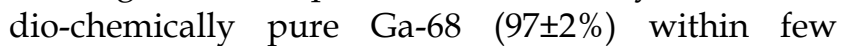
minutes ready for on-site labeling with high overall product yields. The single most imperative aspect of PET/CT is its ability to quantify the disease at a molecular level; for a quantitative approach, Ga-68 and PET/CT is clearly superior to gamma-emitting radionuclides and single photon emission computed tomography (SPECT). Since its first clinical use at our centre in July 2004, there has been a tremendous increase in the number of studies with Ga-68, demonstrating its potential to become the Tc-99m for PET/CT.

\section{THERANOSTICS OF NETs}

Neuroendocrine tumors (NETs) are essentially characterized by their endocrine metabolism and a distinct histological pattern. The heterogeneous nature, frequently indolent course and possibility of multiple and variable anatomic sites of the primary tumor make it difficult to evaluate patients with NETs. Most differentiated NETs over-express somatostatin receptors (SSTRs). Somatostatin receptor scintigraphy (SRS) using In-111 penetreotide has been used for the diagnosis of NETs. ${ }^{3,4}$ However, the development of novel somatostatin analogs for labeling with Ga-68 and also with therapeutic radionuclides like Lu-177 and Y-90 has enabled highly specific targeting of NETs for theranostics. The Ga-68 labeled DOTA-peptides binding to SSTRs with high affinity, which have been used in the clinical setting, are DOTA-D-Phe1-Tyr3-octreotide (DOTATOC), DOTA1-Nal3-octreotide (DOTANOC) and DOTA-D-Phe1-Tyr3-Thr8-octreotide (DOTATATE). Previous studies have established the efficacy of these Ga-68 labeled DOTA-peptides in the diagnosis of NETs. $5-9$

In a recent study in normal human tissues, the maximum SUV (SUVmax) of Ga-68 DOTATOC has been related to the expression of SSTR2 at the level of mRNA. This was a highly interesting observation demonstrating the real power of molecular imaging, and may improve diagnostics, monitoring and therapy of SSTR-expressing tumors on a molecular basis. ${ }^{10}$ Our own recent study provided for the first time, the proof of concept of the utility of Ga-68 somatostatin receptor PET/CT (SSTR PET/CT) for quantification of the SSTR density on tumor cells, showing a close correlation between maximum SUV and immunohistochemical scores used for the quantitative assessment of the density of subtypes of SSTRs in NET tissue. ${ }^{11}$

Ten years ago, Hofmann et al. have shown for the first time that Ga-68 DOTATOC is superior to In-111 Octreotide SPECT (where at that time CT was taken as the reference for comparison) in detecting upper abdominal metastases. ${ }^{5}$ In a recent study, Ga-68 DOTATOC PET/CT was proven to be far superior to In-111 DTPA-OC in the detection of NET metastases in the skeleton and other organs. ${ }^{6}$ In another study using Ga-68 DOTATOC PET, which is in full agreement with our own observations, the accuracy of Ga-68 DOTATOC PET (96\%) was found to be significantly higher than that of CT (75\%) and In-111 DOTATOC SPECT (58\%) on a patient basis. ${ }^{7}$ In 32/88 patients, Ga-68 DOTATOC PET was true positive whereas SPECT results were false negative; in addition, PET was able to detect more lesions than SPECT and CT. The authors also reported that for the staging of patients, PET was better than CT or SPECT as it picked up more lesions in lymph nodes, liver and bones. Overall, PET provided clinically relevant additional information in $14 \%$ of the patients when compared to SPECT and in $21 \%$ of patients when compared to CT. Kowalski et al. described the superiority of Ga-68 DOTATOC PET over In-111 octreotide scan, especially in detecting small tumors or tumors bearing only a low density of SSTRs. ${ }^{12}$ In a larger subgroup of patients $(n=90)$ with pathologically confirmed NETs, a comparison between Ga-68 DOTANOC PET/CT and conventional imaging (CI CT and endoscopic ultrasound) showed the superiority of PET/CT over CI. ${ }^{8}$ Our experience after more than 5,800 SSTR PET/CT studies performed at the Zentralklinik Bad Berka is that Ga-68 SSTR PET is able to detect many lesions which are not routinely detected by $\mathrm{CT}$, magnetic resonance imaging (MRI), skeletal scintigraphy or ultrasonography (Fig. 1).

Ga-68 SSTR PET/CT provides a "one-stop shop" whole-body investigation of NETs (Fig. 2) for staging, including evaluation of liver, lymph nodes, bone, lung, brain and other possible tumor sites. Apart from gastroenteropancreatic NETs, SSTR PET/CT with Ga-68 labeled somatostatin analogs has also been envisaged to have a potential role in small cell lung cancer as this tumor is known to express SSTRs. ${ }^{13,14}$ In a bi-centric study, our group has checked the role of SSTR PET/CT in the detection of unknown primary (CUP) NETs in which Ga-68 DOTANOC PET/CT was found to be highly superior to In-111 Octreoscan and can play a major role in the management of patients 
with CUP-NET. ${ }^{15}$ SSTR PET/CT is particularly of theranostic value in the evaluation of the receptor status before peptide receptor radionuclide therapy (PRRNT), or before therapy with somatostatin ana$\operatorname{logs}$ (e.g. octreotide or lanreotide) to determine the receptor density semi-quantitatively by the measurement of SUV (Standardized Uptake Values). ${ }^{16}$ Another major advantage of Ga-68 SSTR PET/CT is in the follow up and evaluation of molecular response (MORE) to therapy by assessing the molecular tumor volume and by quantification of the SSTR density in vivo before and after PRRNT (Fig. 3). ${ }^{14}$ It also helps in re-staging, e.g. in patients with rising tumor markers (chromogranin A, serotonin, calcitonin, glucagon) and for detection of recurrences.

Ga-68 DOTATOC PET has been found to be superior to F-18 FDG PET in the detection of well-differentiated NETs, demonstrating 57/63 lesions in 15 patients, as compared to only $43 / 63$ de- tected by FDG PET. ${ }^{17}$ In malignant neural crest tumors (pheochromocytoma, paraganglioma, and medullary thyroid cancer), a direct comparison with I-123 MIBG imaging showed the superiority of Ga-68 DOTATATE PET/CT in terms of sensitivity. ${ }^{18}$ An intra-individual study comparing the diagnostic efficacy of Ga-68 DOTANOC and Ga-68 DOTATATE demonstrated that Ga-68 DOTANOC may be superior to Ga-68 DOTATATE in certain cases. ${ }^{19}$

Receptor PET/CT using Ga-68 labeled somatostatin analogs enables molecular imaging of NETs and their metastases with very high diagnostic sensitivity and specificity. It provides quantitative and reproducible data (SUV) which can be used for selecting patients for PRRNT and evaluation of therapy response. Among other advantages are fast protocol (60-90 min.), low radiation burden (10-12 mSv), flexibility in daily use and lower cost than octreotide scintigraphy.

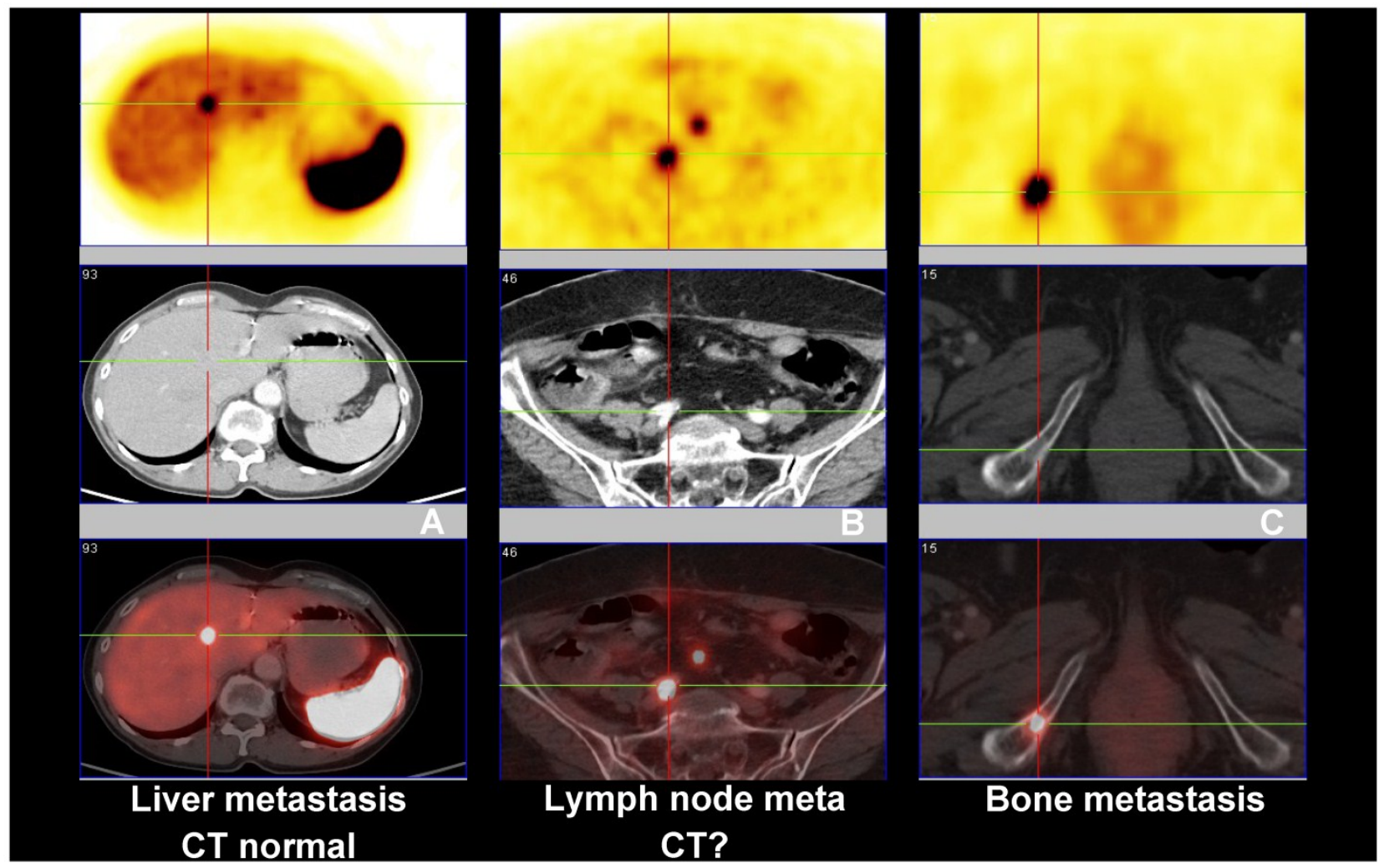

Fig. I: Comparison between Ga-68 SSTR PET/CT (PET images upper row and fused PET/CT lower row), and CT (middle row) showing (A) liver metastasis with normal CT, (B) lymph node metastasis with inconclusive CT findings and (C) bone metastasis seen on both PET and CT images. 


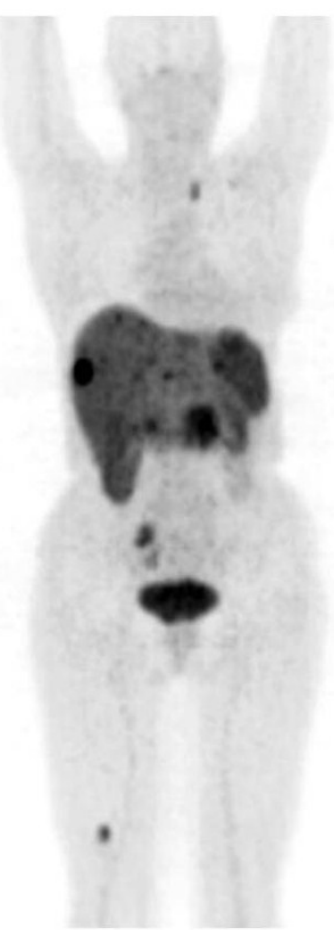

A

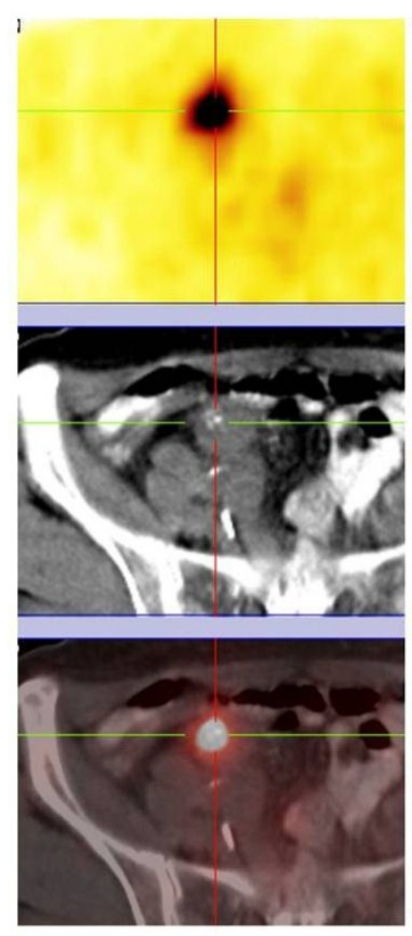

B

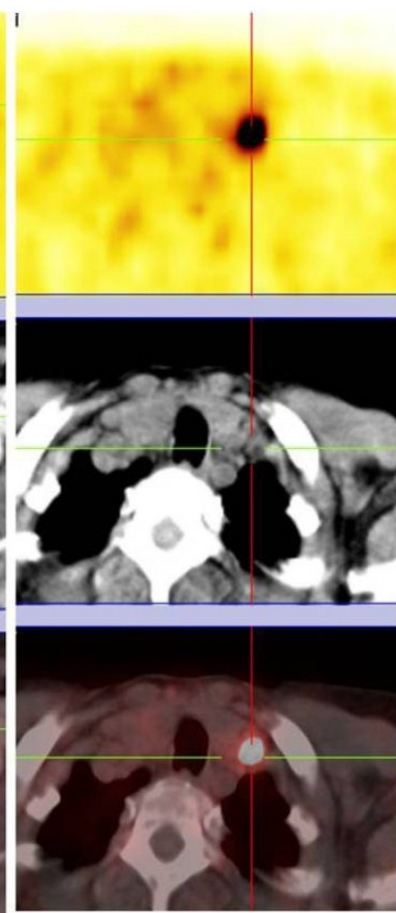

C

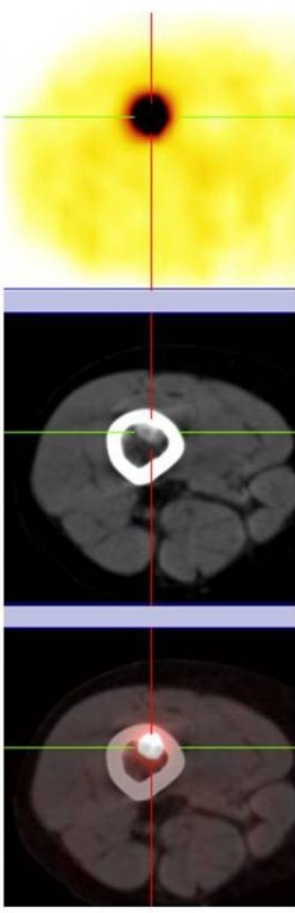

D

Fig. 2: Whole body "one-stop shop" diagnosis with receptor-PET/CT (A - MIP; B, C and D - PET, CT and fused images) using Ga-68 DOTATOC showing primary neuroendocrine tumor in the ileum (B) with lymph node $(C)$ \& bone metastases (D).
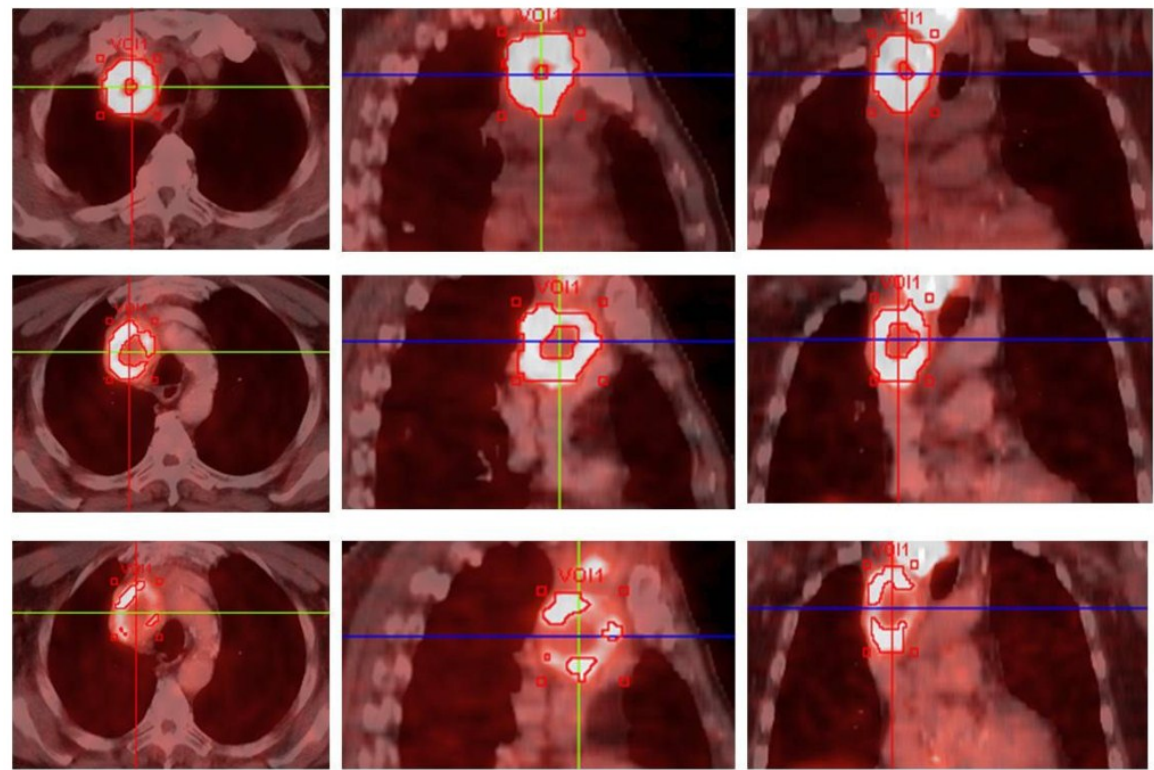

3 months after 2nd PRRNT:

$M T V=28.2 \mathrm{ml}$

SUV $=6.3$

$\mathrm{MTI}=177.7$

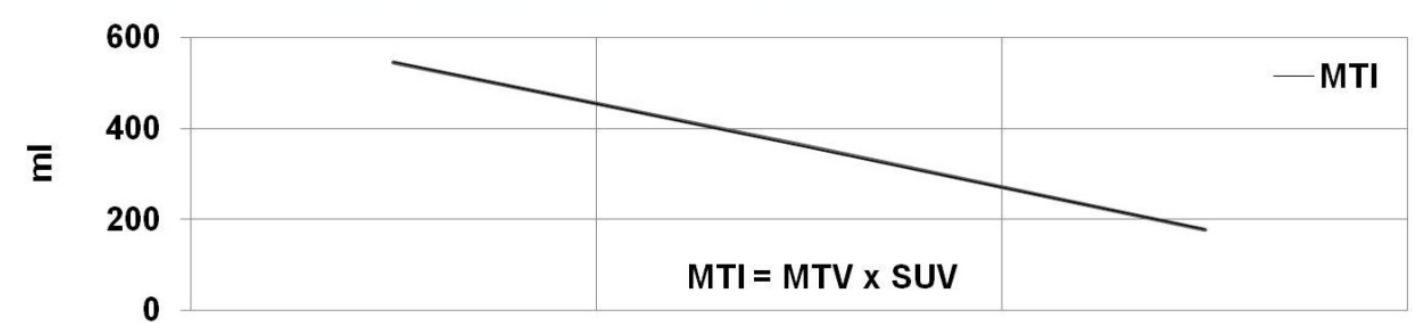

Fig. 3: Monitoring of molecular response to peptide receptor radionuclide therapy with Lu- I77/Y-90 labeled somatostatin analogs by quantification of somatostatin receptor density using Ga-68 somatostatin receptor PET/CT. 
The strong expression of SSTR2 by NETs also enables PRRNT, the molecular internal radiation therapy of NETs. The significant points to take into account concerning PRRNT are patient selection, appropriate choice of peptide and radionuclide, kidney protection (lysine, arginine, gelofusine), tumor and organ dosimetry (post-treatment scans) and monitoring of toxicity (follow-up). In our hospital which was certified as ENETS Center of Excellence in March 2011, a dedicated multidisciplinary team of experienced NET specialists is responsible for the management of NET patients (over 1,000 patient visits per year).

Patient selection for PRRNT is based on the Bad Berka Score (BBS) which takes into accounts the following clinical aspects and molecular features:

1. SUV on receptor PET/CT (for referrals: Krenning's score on OctreoScan) for determining SMS receptor density

2. Renal function - glomerular fil-tration rate (GFR) measured by Tc-99m DTPA and tubular ex-traction rate (TER) measured by Tc-99m MAG3 as well as serum creatinine and blood urea nitrogen (BUN), and elimination kinetics

3. Hematological status (blood counts)

4. Liver involvement

5. Extra-hepatic tumor burden

6. Ki-67 index / tumor grade

7. F-18 fluorodeoxyglucose (FDG) PET/CT status (glucose hy-permetabolism of tu-mors/metastases)

8. Tumor dynamics (doubling time, new lesions)

9. Karnofsky performance score (KPS) or ECOG scale

10. Weight loss

11. Time since first diagnosis

12. Functional activity of tumor

13. Previous therapies

The therapy plan for each patient is individualized. Frequent therapy cycles (4-6 and up to 8), applying low or intermediate doses of radioactivity are suitable for these relatively slow-growing tumors ("long term low dose, not short term high dose concept $\left.^{\prime \prime}\right)$. For kidney protection, patients are well hydrated and receive an amino acid infusion containing lysine and arginine given intravenously for 4 hours beginning 30 minutes before PRRNT. Before each new treatment cycle, restaging is performed by morphologic (CT/MRI) and molecular imaging (Ga-68 SSTR PET/CT, in selected cases F-18 FDG or F-18 fluoride PET/CT studies are additionally performed), blood chemistry and tumor markers (chromogranin A, ser- otonin, specific hormones). Renal function is serially determined by Tc-99m MAG3 scan/TER and by Tc-99m DTPA (GFR) measurements. All data are entered in a prospective structured database (programmed in ACCESS and containing 284 items per patient). Another very important aspect of personalized medicine and THERANOSTICS is dosimetry (Fig. 4). Estimation of tumor and normal organ doses (MIRD/OLINDA) performed after PRRNT (using Lu-177 labeled somatostatin analogs DOTATATE or DOTATOC) is important to ensure that maximum dose is delivered to the tumors and therefore optimizing an individualized treatment protocol. ${ }^{20}$

In patients with progressive NETs of non-pancreatic origin and pancreatic NETs (pNETs), tumor response after a mean follow-up of 2 years was as follows: after 3 PRRNT cycles, complete remission (CR), partial remission (PR), and minor response (MR) were seen in $52 \%$ of patients with pNETs (and in $48 \%$ in non pancreatic NETs); disease was stabilized in $39 \%$ of pNETs and in $45 \%$ of patients with non pancreatic NETs (Fig. 5). Thirty-six patients with advanced disease died of progressive disease (PD). Objective tumor responses (including improvement of clinical symptoms) were seen in $93 \%$ (91\% pNETs) of the patients. Significant hematological toxicity (mainly erythrocytopenia, rarely neutropenia, and thrombocytopenia) occurred in less than $15 \%$ of all patients. Myelodysplastic syndrome (MDS) developed in 5 patients (all of them received also chemotherapy before). End stage renal insufficiency was not observed in any of the patients with normal kidney function before PRRNT. In most patients receiving Lu-177 DOTATATE alone ( $\mathrm{n}=417$ cycles), serum creatinine and TER/GFR did not change significantly. Therefore, the probability and magnitude of renal toxicity can be significantly reduced (or completely avoided) when PRRNT is administered in fractionated doses in patients without any pre-existing risk factors and under appropriate nephroprotection. Chemotherapy, diabetes mellitus, hypertension, Hedinger's syndrome, and cachexia were identified as the risk factors for nephrotoxicity after PRRNT. ${ }^{21}$

We also have treated patients with progressive metastases of NETs and with a single functional kidney (24 patients). None of these patients showed grade 3 or 4 nephrotoxicity. PRRNT resulted in partial remission in $36 \%$ and stable disease in $36 \%$ of the patients, $28 \%$ had PD. 14 had grade 1 erythrocytopenia, 3 had grade 1 leucocytopenia and 3 had grade 1 thrombocytopenia. In 2009, we have given fractionated low dose PRRNT to 2 patients on hemodialysis due to end-stage renal insufficiency (to the best of our knowledge, this was the first ever worldwide experi- 
ence with such a condition). No significant hematotoxicity was observed in the two patients on dialyses and both showed a good clinical and objective therapy repsonse. ${ }^{22}$

\section{A}
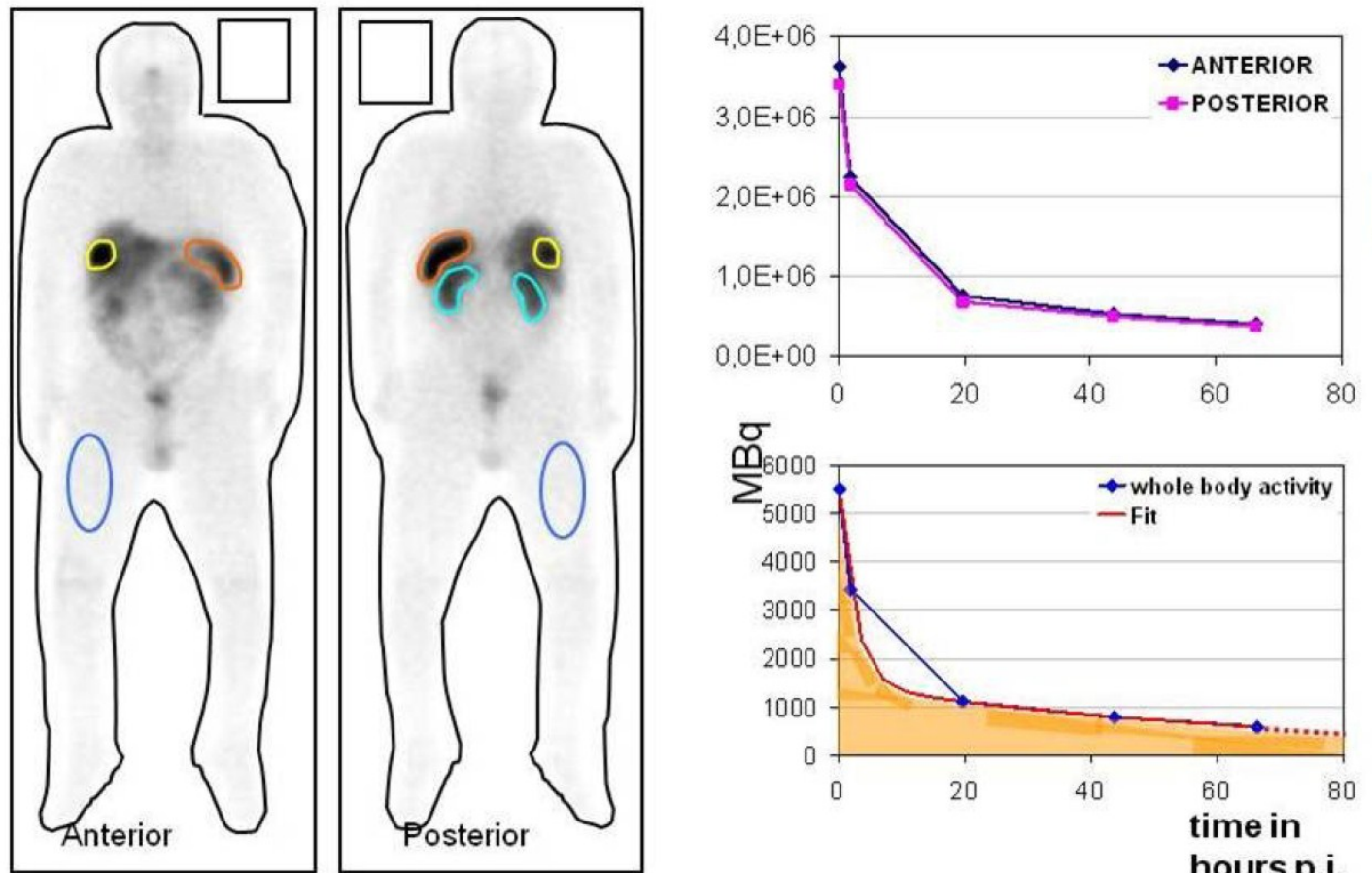

B

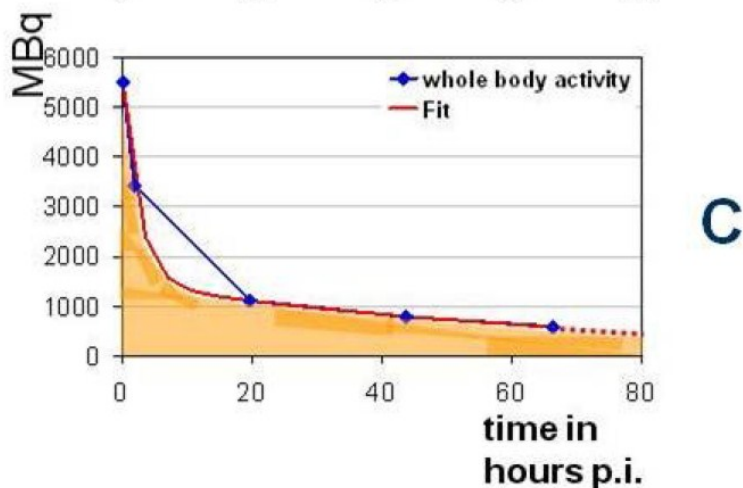

Fig. 4: The Bad Berka Dose protocol - Analysis of regions of interest around whole body, tumor and normal organs in anterior and posterior planar whole body post-therapy (Lu-I77 DOTA-somatostatin analogs) scans at 5 time intervals (A), determination of time activity curves in anterior and posterior scans (B) and using the geometric mean, fitting of the time-activity curves to an exponential function to obtain the residence time of the radiopharmaceutical in the region of interest.

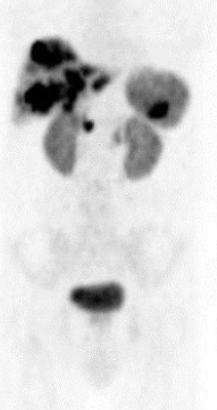

Pre PRRNT

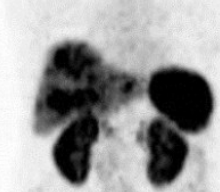

3 Months post PRRNT
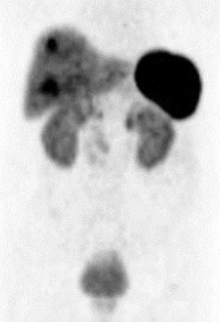

9 Months post PRRNT

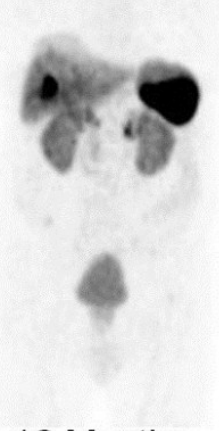

12 Months post PRRNT

Fig. 5: VIPoma patient with Verner Morrison syndrome (severe watery diarrhoea, hypokalemia), high dose octreotide (Sandostatin LAR) prior to PRRNT. After administration of one single cycle of 5 GBq Y-90 DOTATATE, there was no need of octreotide after 3 months, I5 $\mathrm{kg}$ weight gain and significant reduction of tumor burden (partial remission. After follow up of I year, the liver and kidney functions were normal and only single liver metastasis remained. 


\section{Nephrotoxicity evaluation-Y-90 vs. Lu-177 vs. DUO PRRNT}

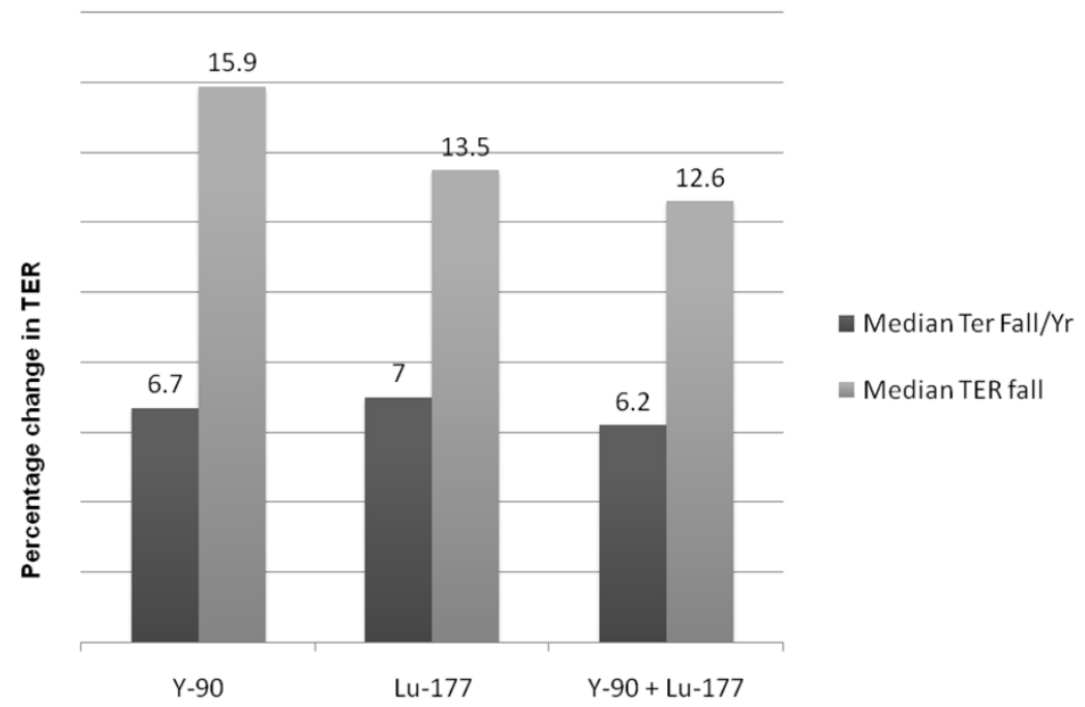

Fig. 6: Long term effect on renal function depending on the radionuclide used for PRRNT.

The Bad Berka neuroendocrine tumor center (BBNETC) group was the first also to use Y-90 DOTATATE, and in a large patient group, Lu-177 DOTATOC in progressive NETs, non-responsive to octreotide/interferon treatment or chemotherapy. An important influence on the decision of the choice of radionuclide is the size of tumors. More commonly, patients present with tumors of various sizes and inhomogeneous distribution of somatostatin receptors. The use of a combination of radionuclides Lu-177 and Y-90 takes this heterogeneity into account. Sequential administration of Y-90 and Lu-177 labeled analogs also is similarly helpful for the treatment of larger tumors, followed by treatment of smaller metastases respectively in further treatment cycles. The BBNETC group pioneered the systematic use of Y-90 and Lu-177 DOTATATE (DUO-PRRNT) in sequence and concurrently, as well as the intra-arterial use of Y-90 DOTATATE and DOTATOC. Lu-177 DOTATATE was predominantly used for small metastases or in patients with impaired renal or hematological function. Long term follow-up of up to 7 years after DUO PRRNT showed no significant grade 3 or grade 4 nephrotoxicity attributed to concurrent or sequential DUO PRRNT. The median fall in tubular extraction rate (TER) was lesser in patients undergoing DUO PRRNT than in those undergoing PRRNT with Lu-177 or Y-90 alone (Fig. 6). The results of a study by $\mathrm{Ku}-$ nikowska et al also indicated that tandem PRRNT (with Y-90/Lu-177 DOTATATE) provided longer overall survival than with a single radioisotope (Y-90 DOTATATE) and the safety of both methods was comparable. ${ }^{23}$

Thus, in patients with progressive NETs, fractionated, personalized PRRNT with lower doses of radioactivity given over a longer period of time (Bad Berka Protocol) is effective even in advanced cases and results in excellent therapeutic responses. Up to 8 cycles of PRRNT, given over several years were tolerated very well by most patients. Severe hematological and/or renal toxicity can be avoided or reduced. Quality of life can be significantly improved. Though cure is rarely possible, excellent palliation with significant improvement of symptoms can be achieved by PRRNT. In addition, neoadjuvant PRRNT could be administered in cases of inoperable NET so as to render the tumor operable by inducing radiation induced necrosis. ${ }^{24,25}$

A recent analysis of 416 patients (all NET subtypes) treated at the BBNETC showed a median overall survival from the time of first diagnosis of 210 months (Rotterdam data 128 months) and a median survival after 1st PRRNT of 59 months (Rotterdam data 46 months). ${ }^{26}$ Our experience confirms a previous report from the Rotterdam group that - compared with historical controls - there is a benefit in overall survival from time of diagnosis of several years. In progressive NETs, sequential (DUO) or concurrent (TANDEM) PRRNT with Lu-177 and Y-90 has been found to be most effective (highest CR / PR / SD rate).

However, PRRNT should only be performed at specialized centers as NET patients need highly individualized interdisciplinary treatment and long term 
care. $^{27}$ Use of intra-arterial PRRNT (> 50 treatments already performed up to now) is more effective for selectively targeting liver metastases and large, inoperable primary tumors. PRRNT can be effectively combined with transarterial chemoembolization (TACE), radio-frequency ablation (RFA), chemotherapy (e.g. using Capecitabine, Temazolomide or Doxorubicin), kinase inhibitors (e.g. Everolimus, Sunitinib or Sorafenib) or possibly with antibodies (e.g. Bevacizumab).

\section{THERANOSTICS OF NON-NETs}

A number of novel Ga-68 labeled tracers (Table 1) have recently become available for THERANOSTICS. ${ }^{28}$ This also includes non-peptide tracers such as the biphosphonate-based agent BPAMD - ((4-\{[bis-(phosphonomethyl)) carbamoyl]methyl\}-7,10 bis(carboxymethyl)-1,4,7,10tetraazacyclododec-1-yl)acetic acid. ${ }^{29}$ PET/CT with Ga-68 BPAMD using the readily-available generator-produced radionuclide is more sensitive and provides for high resolution imaging, with concurrent whole body CT for anatomical correlation.

The Bad Berka group was also the first to use in humans, a Ga-68 labeled gastrin releasing peptide receptor (GRP-R) selective bombesin analogue AMBA (DO3A-CH2CO-G-4-aminobenzoyl-Q-W-A-V-G-H-LM-NH2) and the GRP-R antagonist demobesin for imaging of metastatic breast, lung and prostate cancers. ${ }^{30}$ Many other Ga-68 radiopharmaceuticals have been used by our group as first in human studies, such as Ga-68 labeled macroaggregates (MAA) for lung perfusion PET/CT ${ }^{31}$, Ga-68 DOTA- alpha-MSH (melanocyte stimulating hormone) in a patient with metastatic ocular melanoma ${ }^{32}$, and Ga-68 labeled affibody molecule targeting HER2 (human epidermal growth factor receptor 2$)^{33}$. Our experience confirms that Ga-68 is a very useful, practical and affordable radionuclide for high resolution PET/CT imaging and holds great promise for the future.

Lu-177-BPAMD has been used in patients with widespread, painful skeletal metastases, presenting with progressive disease and refractory to conventional treatment (Fig. 7). ${ }^{34}$ Dosimetry showed that due to the very long half life of the radiopharmaceutical in the metastases ( $>80$ hours), tumor doses delivered were high, ranging from $2.4-209 \mathrm{mGy} / \mathrm{MBq}$ (wide range due to different size of the lesions). A significant reduction in osteoblastic activity of the bone metastases was seen on the follow up PET/CT using Ga-68 BPAMD or F-18 fluoride. The treatment was very well tolerated by all patients without any significant acute adverse effects. There were only mild to moderate changes in blood cell counts, and no significant alterations in serum creatinine/BUN or other lab parameters were observed. The first ever Lu-177 demobesin therapy was also administered in 2009. In continuum, a novel GRP-R antagonist labeled with Lu-177 was administered in a patient with metastatic prostate cancer. Noteworthy amongst the other therapeutic radiopharmaceuticals (Table 2) is also the first antiangiogenic radionuclide therapy using Lu-177 labeled RGD (arginine-glycine-aspartic acid). ${ }^{35}$

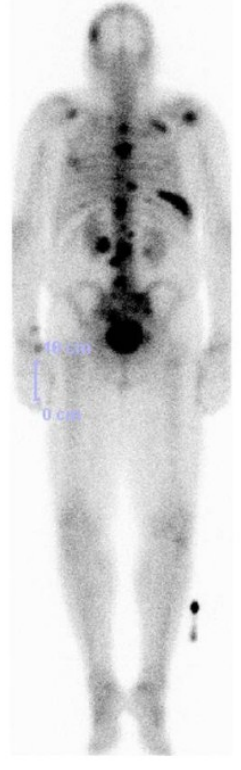

$30 \mathrm{~min}$

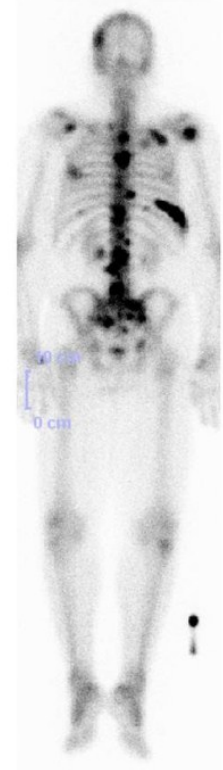

$3 \mathbf{h}$

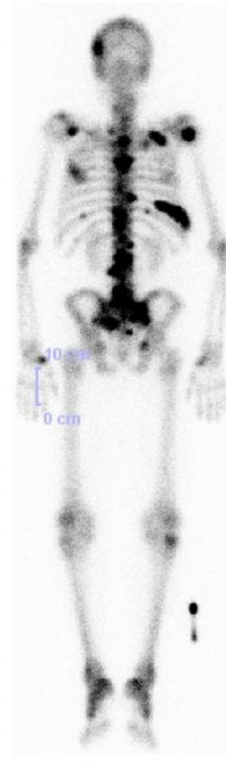

$21 \mathrm{~h}$
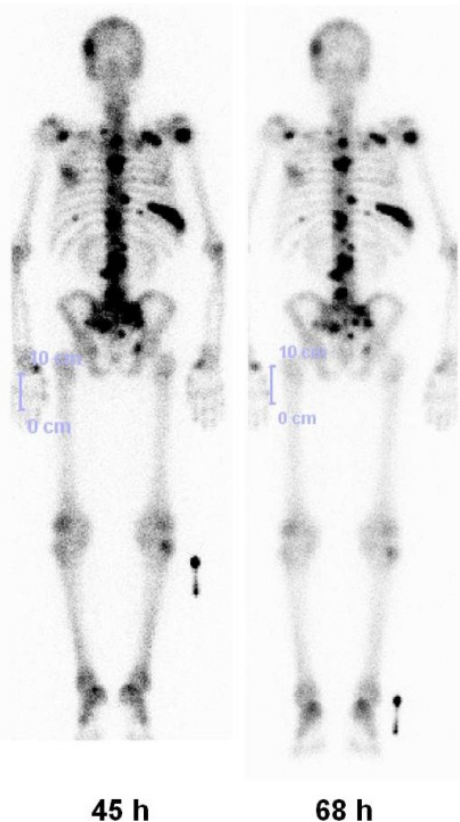

Fig. 7: Lu-I77 BPAMD post-therapy planar images (posterior view) in a patient of metastatic prostate cancer at 5 time intervals for dosimetry, showing high uptake in skeletal metastases with high contrast and long effective half life (retention), and rapid renal clearance. 
Table I. Diagnostic radiopharmaceuticals first used at the Bad Berka neuroendocrine tumor and THERANOSTICS center

\begin{tabular}{|c|c|c|c|}
\hline Radiopharmaceutical & $\begin{array}{l}\text { First use } \\
\text { (month/year) }\end{array}$ & Principle & Clinical indication \\
\hline Ga-68 DOTANOC & $07 / 2004$ & SSTR targeting (agonist) & NETs \\
\hline * Ga-68 gastrin & $12 / 2006$ & Gastrin receptor targeting & Medullary carcinoma of thyroid \\
\hline * Ga-68 AMBA & $08 / 2006$ & $\begin{array}{l}\text { Bombesin analogue - GRP and NMB } \\
\text { receptor targeting }\end{array}$ & Carcinomas of breast, prostate, colon \\
\hline * Ga-68 tyrosine & $08 / 2008$ & Amino acid uptake by tumour cells & Brain tumors (Gliomas) \\
\hline * Ga-68 BPAMD & $02 / 2009$ & $\begin{array}{l}\text { Biphosphonate - Binding to hydrox- } \\
\text { yapatite structure of bone }\end{array}$ & Skeletal metastases \\
\hline * Ga-68 MAA & $09 / 2008$ & $\begin{array}{l}\text { Mechanical trapping of MAA } \\
\text { (Macroaggregated albumin) in the } \\
\text { capillary bed }\end{array}$ & $\begin{array}{l}\text { Pulmonary thromboembolism, visualization of the } \\
\text { perfusion pattern before selective intra-arterial } \\
\text { therapy (e.g. internal radiation using Y-90 for hepatic } \\
\text { metastases) }\end{array}$ \\
\hline * Ga-68 glucose & $05 / 2009$ & Tumor hypermetabolism & Similar to the use of 18F-FDG in tumors \\
\hline * Ga-68 demobesin & $07 / 2009$ & GRP receptor antagonist & Carcinomas of breast, prostate, colon \\
\hline * Ga-68 JR10 & $05 / 2009$ & SSTR targeting (antagonist) & NETs \\
\hline * Ga-68 alpha-MSH & $05 / 2010$ & $\begin{array}{l}\text { Cell surface receptor targeting of } \\
\text { melanocytes }\end{array}$ & Melanoma \\
\hline * Ga-68 sarabesin-6 & $08 / 2011$ & GRP receptor antagonist & Carcinomas of breast, prostate, colon \\
\hline * Ga-68 RGD & $09 / 2011$ & $\begin{array}{l}\text { Integrin/ } \\
\text { alpha-v beta- } 3 \text { targeting (antagonist) }\end{array}$ & $\begin{array}{l}\text { Investigation and monitoring of neoangiogenesis } \\
\text { and invasiveness of cancers like breast, colon, pros- } \\
\text { tate, lung etc. }\end{array}$ \\
\hline * Ga-68 HER-2 affibody & $12 / 2005$ & HER-2 targeting & $\begin{array}{l}\text { Prognostication in carcinoma breast (investigation of } \\
\text { HER-2 status before herceptin therapy) }\end{array}$ \\
\hline * Ga-68 SHAL & $05 / 2010$ & $\begin{array}{l}\text { Selective high affinity ligand for B-cell } \\
\text { lymphoma }\end{array}$ & B-cell Lymphoma \\
\hline * Tc-99m demobesin & $11 / 2009$ & GRP receptor antagonist & Carcinomas of breast, prostate, colon \\
\hline * Scandium-44 DOTATOC & 02/2009 & SSTR targeting (agonist) & Pre-therapeutic dosimetry before PRRNT in NETs \\
\hline
\end{tabular}

(DOTANOC - DOTA-1-Nal3-octreotide; SSTR - somatostatin receptor; NETs - neuroendocrine tumors; * - first in human study; AMBA DO3A-CH2CO-G-4-aminobenzoyl-Q-W-A-V-G-H-L-M-NH2; GRP - Gastrin releasing peptide; NMB - Neuromedin B; BPAMD -

((4-\{[bis-(phosphonomethyl)) carbamoyl]methyl\}-7,10 bis(carboxymethyl)-1,4,7,10-tetraazacyclododec-1-yl)acetic acid; MAA - macro aggregated albumin; FDG - fluorodeoxyglucose; JR 10 - somatostatin receptor antagonist (Courtesy Helmut Maecke, Basel, Switzerland); MSH - melanocyte stimulating hormone; RGD - arginine-glycine-aspartic acid; HER-2 - human epidermal growth factor receptor 2; SHAL selective high affinity ligand; DOTATOC - DOTA-D-Phe1-Tyr3-octreotide; PRRNT - peptide receptor radionuclide therapy)

Table 2. Therapeutic radiopharmaceuticals first used at Bad Berka THERANOSTICS Center

\begin{tabular}{lll}
\hline Radiopharmaceutical & First use (month/year) & Clinical indication \\
\hline $\begin{array}{l}\text { Lu-177 DOTATATE } \\
\text { * Combined Y-90 and Lu-177 labeled somatostatin analogs }\end{array}$ & $08 / 2004$ & PRRNT in NETs \\
$\begin{array}{l}\text { * Systematic use of sequential Y-90 and Lu-177 labeled } \\
\text { somatostatin analogs }\end{array}$ & & TANDEM-PRRNT in NETs \\
* Intra-arterial Y-90 DOTATATE & & Selective internal radiation therapy in NETs \\
Lu-177 AMBA & & Radionuclide therapy of metastatic carcinoma breast \\
* Lu-177 RGD & Anti-angiogenesis therapy \\
* I-131 Phenylalanine (ACD-101) & Endoradiotherapy in brain tumor (glioma) \\
* Lu-177 BPAMD & $04 / 2009$ & $\begin{array}{l}\text { Radionuclide therapy and pain palliation of skeletal } \\
\text { metastases in carcinoma prostate }\end{array}$ \\
* PRRNT with Lu-177 DOTATATE in a patient on hemodi- & $06 / 2009$ & First PRRNT in NET with end stage renal disease \\
alysis & & Radionuclide therapy in carcinoma breast \\
* Lu-177 demobesin & $12 / 2009$ & Radionuclide therapy in carcinoma prostate \\
* Lu-177 sarabesin-6 & $04 / 2011$ &
\end{tabular}




\section{FUTURE PERSPECTIVES}

As we move towards personalized medicine, the diagnostic information obtained from PET/CT must be improved, i.e., by fast and routine quantification of lesions. The Bad Berka Molecular Imaging Tool (BBQ-MIT) has been developed by us based on the cognition network language (CNL), provided by Definiens AG (Munich, Germany). BBQ-MIT is an automatic, user-independent routine for segregation and quantification of neoplastic lesions in molecular PET/CT DICOM sets (Fig. 8). This prototype routine built on CNL for PET/CT images enables automatic analysis of lesions, e.g. by calculating SUV, molecular tumor volume (MTV), molecular tumor diameter (MTD), molecular tumor index (MTI = SUV $x$ MTD), whole-body and organ tumor burden and many other parameters. It seems especially promising for shortening the analysis time for reading a PET scan with many tumor lesions, improving reproducibility, as well as increasing the sensitivity in lesion detection. We firmly believe that BBQ-MIT is a definite step forward and should set a trend towards the fast and accurate analysis of serial PET/CT, allowing monitoring of tumor response and assessment of therapy effect early in the course of therapy, thus enabling effective personalized patient management.

Also promising prospects in the THERANOSTICS of NETs include the use of fourth generation peptides, SSTR antagonists (these peptides may be superior to agonists due to higher receptor density on tumor cells), somatostatin analogs targeting all known SSTR subtypes (except SSTR4), and therefore a wider tumor spectrum, and targeting of multiple receptors. A more accurate and truly individualized approach to dosimetry would be with development of newer methods for 3D calculation of dose. Pre-therapeutic organ and tumor dosimetry using receptor PET/CT can be accomplished using longer lived positron emitters, e.g. Sc-44, Y-86 or Cu-64 and comparison of these results with those obtained from Ga-68, are needed. The appropriate peptide and radionuclide for individualized therapy ("personalized medicine") can be then selected by pre-therapeutic measurement of organ and tumor doses. The first human study using the longer-lived, generator-derived, trivalent metallic positron emitter Scandium-44 (Sc-44) coupled to DOTATOC was performed in Bad Berka in 2009. Sc-44 has a half life of 3.9 hrs and is derived from a Titanium-44/Scandium-44 generator, which has a half life of 60 years. ${ }^{36,37}$ This may inspire the development of new radiopharmaceuticals for covering longer imaging periods.

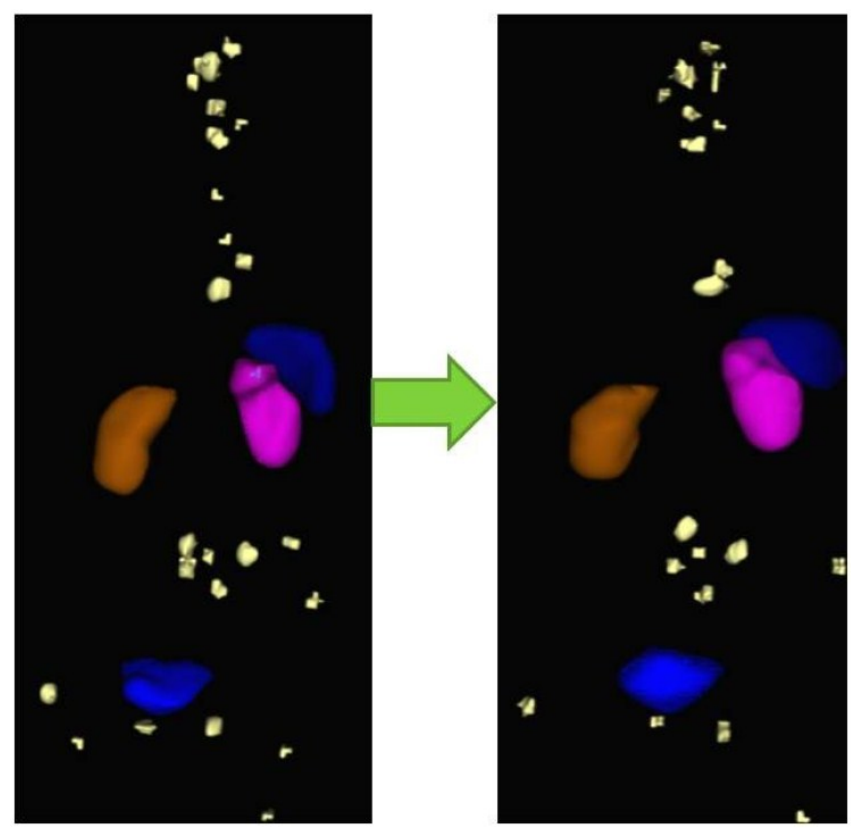

Fig. 8: Example of monitoring therapeutic response by comparison of pre-PRRNT (left) and post-PRRNT Ga-68 somatostatin receptor PET/CT DICOM sets using BBQ-MIT, an automatic, rapid, user-independent routine for segregation and quantification (SUV and tumor volumes) of neoplastic lesions by determine the lesions' borders.

\section{ACKNOWLEDGMENTS}

The authors are very grateful to Helmut Mäcke for developing the technique of DOTA-conjugated somatostatin analogs, for providing the activity (Y-90 DOTATOC) for the first patient treatment in Germany, a number of peptides for clinical trials and for many fruitful scientific discussions over the years. We are indebted to Frank Rösch for help in routinely establishing the Ge-68/Ga-68 generator system in our center for labeling of peptides for receptor PET/CT studies; and to many other colleagues and our staff for continuous support. We would also like to thank Vikas Prasad for his valuable contribution in the analysis of some of the PRRNT results (e.g. renal toxicity, see figure 6) at our center.

This publication is dedicated to Dr. Gustav Hör, Professor Emeritus, University of Frankfurt/Main, on the occasion of his 80th birthday.

\section{Competing Interests}

The authors have declared that no competing interest exists.

\section{References}

1. Rösch F, Baum RP. Generator-based PET radiopharmaceuticals for molecular imaging of tumours: on the way to THERANOSTICS. Dalton Trans. 2011; 40: 6104-11. 
2. Zhernosekov KP, Filosofov DV, Baum RP, et al. Processing of generator-produced ${ }^{68} \mathrm{Ga}$ for medical application. J Nucl Med. 2007; 48: 1741-8

3. Lamberts SWJ, Reubi JC, Krenning EP. Somatostatin and the concept of peptide receptor scintigraphy in oncology. Semin Oncol. 1994; 21: 1-5.

4. Krenning EP, Kwekkeboom DJ, de Jong M, et al. Essentials of peptide receptor scintigraphy with emphasis on somatostatin analog octreotide. Semin Oncol. 1994; 21: 6-14.

5. Hofmann $\mathrm{M}$, Maecke $\mathrm{H}$, Borner $\mathrm{R}$, et al. Biokinetics and imaging with the somatostatin receptor PET radioligand (68)Ga-DOTATOC: preliminary data. Eur J Nucl Med Mol Imaging. 2001; 28: 1751-7.

6. Buchmann I, Henze M, Engelbrecht S, et al. Comparison of 68Ga-DOTATOC PET and 111In-DTPA-OC (Octreoscan) SPECT in patients with neuroendocrine tumours. Eur J Nucl Med Mol Imaging. 2007; 34: 1617-26.

7. Gabriel M, Decristoforo C, Kendler D, et al. 68Ga-DOTA-Tyr3-octreotide PET in neuroendocrine tumors: comparison with somatostatin receptor scintigraphy and CT. J Nucl Med. 2007; 48: 508-18.

8. Ambrosini V, Campana D, Bodei L, et al. 68Ga- DOTA-NOC PET/CT clinical impact in patients with neuroendocrine tumors. J Nucl Med. 2010; 51: 669-73.

9. Srirajaskanthan R, Kayani I, Quigley AM, et al. The role of $68 \mathrm{Ga}-$ DOTATATE PET in patients with neuroendocrine tumors and negative or equivocal findings on 111In- DTPA-octreotide scintigraphy. J Nucl Med. 2010; 51: 875-82.

10. Boy C, Heusner TA, Poeppel TD, et al. (68)Ga-DOTATOC PET/CT and somatostatin receptor (sst1-sst5) expression in normal human tissue: correlation of sst2 mRNA and SUV(max). Eur J Nucl Med Mol Imaging. 2011; 38: 1224-36.

11. Kaemmerer D, Peter L, Lupp A, et al. Molecular imaging with ${ }^{68} \mathrm{Ga}$-SSTR PET/CT and correlation to immunohistochemistry of somatostatin receptors in neuroendocrine tumours. Eur J Nucl Med Mol Imaging. 2011; 38: 1659-68.

12. Kowalski J, Henze M, Schuhmacher J, et al. Evaluation of positron emission tomography imaging using [68Ga]-DOTA-D Phe(1)-Tyr(3)-Octreotide in comparison to [111In]-DTPAOC SPECT. First results in patients with neuroendocrine tumors. Mol Imaging Biol. 2003; 5: 42-8.

13. Baum RP, Prasad V. PET and PET/CT Imaging of Neuroendocrine Tumors. In: Wahl R, ed. Principles and Practice of PET and PET/CT, $2^{\text {nd }}$ ed. Philadelphia: Lippincott Williams \& Wilkins; 2008: 411-37.

14. Baum RP, Prasad V, Hommann M, et al. Receptor PET/CT imaging of neuroendocrine tumors. Recent Results in Cancer Research. 2007; 170: 225-42.

15. Prasad V, Ambrosini V, Hommann M, et al. Detection of unknown primary neuroendocrine tumours (CUP-NET) using (68)Ga-DOTA-NOC receptor PET/CT. Eur J Nucl Med Mol Imaging. 2010; 37: 67-77.

16. Prasad V, Baum RP. Biodistribution of the Ga-68 labeled somatostatin analogue DOTA-NOC in patients with neuroendocrine tumors: characterization of uptake in normal organs and tumor lesions. Q J Nucl Med Mol Imaging. 2010; 54: 61-7.

17. Koukouraki S, Strauss LG, Georgoulias V, et al. Evaluation of the pharmacokinetics of (68)Ga-DOTATOC in patients with metastatic neuroendocrine tumours scheduled for (90)Y-DOTATOC therapy. Eur J Nucl Med Mol Imaging. 2006; 33: 460-6.

18. Naji M, Zhao C, Welsh SJ, et al. 68Ga-DOTA-TATE PET vs. 123I-MIBG in identifying malignant neural crest tumours. Mol Imaging Biol. 2011; 13: 769-75.

19. Maecke HR, Hofmann M, Haberkorn U. (68)Ga-labeled peptides in tumor imaging. J Nucl Med. 2005; 46 (Suppl 1): S172-S178.

20. Wehrmann C, Senftleben S, Zachert C, et al. Results of individual patient dosimetry in peptide receptor radionuclide therapy with 177Lu DOTA-TATE and 177Lu DOTA-NOC. Cancer Biother Radiopharm. 2007; 22: 406-16.

21. Prasad V, Hoersch D, Zachert C, et al. Five year follow-up of renal function in neuroendocrine tumor patients treated with 5 or more cycles of peptide receptor radionuclide therapy (PRRNT). J Nucl Med. 2011; 52 (Suppl 1): 299.
22. Prasad V, Kulkarni H, Zachert C, et al. Safety and efficacy of peptide receptor radionuclide therapy (PRRNT) in neuroendocrine tumor patients having single kidney or nonfunctional kidney. J Nucl Med. 2011; 52 (Suppl 1): 1741.

23. Kunikowska J, Królicki L, Hubalewska-Dydejczyk A, et al. Clinical results of radionuclide therapy of neuroendocrine tumours with ${ }^{90} Y$-DOTATATE and tandem ${ }^{90} \mathrm{Y} /{ }^{177} \mathrm{Lu}$-DOTATATE: which is a bettertherapy option? Eur J Nucl Med Mol Imaging. 2011; 38(10): 1788-97.

24. Kaemmerer D, Prasad V, Daffner W, et al. Neoadjuvant peptide receptor radionuclide therapy for an inoperable neuroendocrine pancreatic tumor. World J Gastroenterol. 2009; 15: 5867-70.

25. Sowa-Staszczak A, Pach D, Chrzan R, et al. Peptide receptor radionuclide therapy as a potential tool for neoadjuvant therapy in patients with inoperable neuroendocrine tumours (NETs). Eur J Nucl Med Mol Imaging. 2011; 38: 1669-74.

26. Kwekkeboom DJ, de Herder WW, Kam BL, et al. Treatment with the radiolabeled somatostatin analog [177 Lu-DOTA 0,Tyr3] octreotate: toxicity, efficacy, and survival. J Clin Oncol. 2008; 26: 2124-30.

27. Hörsch D, Grabowski P, Schneider $\mathrm{CP}$, et al. Current treatment options for neuroendocrine tumors. Drugs Today. 2011; 47: 773-86.

28. Pagou M, Zerizer I, Al-Nahhas A. Can gallium-68 compounds partly replace (18)F-FDG in PET molecular imaging? Hell J Nucl Med. 2009; 12: 102-5.

29. Fellner M, Baum RP, Kubícek V, et al. PET/CT imaging of osteoblastic bone metastases with (68)Ga-bisphosphonates: first human study. Eur J Nucl Med Mol Imaging. 2010; 37: 834.

30. Schroeder RP, Müller C, Reneman S, et al. A standardised study to compare prostate cancer targeting efficacy of five radiolabelled bombesin analogs. Eur J Nucl Med Mol Imaging. 2010; 37: 1386-96.

31. Kotzerke J, Andreeff M, Wunderlich G, et al. Ventilation/Perfusion Scans Using Ga-68 Labeled Tracers. World J Nucl Med. 2011; 10: 26-59.

32. Froidevaux S, Calame-Christe M, Schuhmacher J, et al. A gallium-labeled DOTA-alpha-melanocyte- stimulating hormone analog for PET imaging of melanoma metastases. J Nucl Med. 2004; 45(1): 116-23.

33. Baum RP, Prasad V, Müller D, et al. Molecular imaging of HER2-expressing malignant tumors in breast cancer patients using synthetic $111 \mathrm{In}$ - or 68Ga-labeled affibody molecules. J Nucl Med. 2010; 51: 892-7.

34. Baum RP, Kulkarni HR, Schuchardt C, et al. First Clinical Experience using 177Lu-BPAMD for the Treatment of Skeletal Metastases in Prostate Cancer. Eur J Nucl Med Mol Imaging. 2011; 38 (Suppl 2): S225.

35. Ju CH, Jeong JM, Lee YS, et al. Development of a ${ }^{177}$ Lu-labeled RGD derivative for targeting angiogenesis. Cancer Biother Radiopharm. 2010; 25(6): 687-91.

36. Majkowska-Pilip A, Bilewicz A. Macrocyclic complexes of scandium radionuclides as precursors for diagnostic and therapeutic radiopharmaceuticals. J Inorg Biochem. 2011; 105(2): 313-20.

37. Pruszyński M, Loktionova NS, Filosofov DV, et al. Post-elution processing of (44)Ti/ (44)Sc generator-derived (44)Sc for clinical application. Appl Radiat Isot. 2010; 68(9): 1636-41. 